\title{
Updating the theoretical tidal evolution constants: Apsidal motion and the moment of inertia ${ }^{\star}$
}

\author{
A. Claret $^{1,2}$ \\ ${ }^{1}$ Instituto de Astrofísica de Andalucía, CSIC, Apartado 3004, 18080 Granada, Spain \\ e-mail: claret@iaa.es \\ 2 Dept. Física Tórica y del Cosmos, Universidad de Granada, Campus de Fuentenueva s/n, 10871 Granada, Spain \\ Received 3 June 2019 / Accepted 1 July 2019
}

\begin{abstract}
Context. The theoretical apsidal motion constants are key tools to investigate the stellar interiors in close eccentric binary systems. In addition, these constants and the moment of inertia are also important to investigate the tidal evolution of close binary stars as well as of exo-planetary systems.

Aims. The aim of the paper is to present new evolutionary models, based on the MESA package, that include the internal structure constants $\left(k_{2}, k_{3}\right.$, and $\left.k_{4}\right)$, the radius of gyration, and the gravitational potential energy for configurations computed from the pre-mainsequence up to the first ascent giant branch or beyond. The calculations are available for the three metallicities $[\mathrm{Fe} / \mathrm{H}]=0.00,-0.50$, and -1.00 , which take the recent investigations in less metallic environments into account. This new set of models replaces the old ones, published about 15 years ago, using the code GRANADA.

Methods. Core overshooting was taken into account using the mass- $f_{o v}$ relationship, which was derived semi-empirically for models more massive than $1.2 M_{\odot}$. The differential equations governing the apsidal motion constants, moment of inertia, and the gravitational potential energy were integrated simultaneously through a fifth-order Runge-Kutta method with a tolerance level of $10^{-7}$.

Results. The resulting models (from 0.8 up to $35.0 M_{\odot}$ ) are presented in 54 tables for the three metallicities, containing the usual characteristics of an evolutionary model (age, initial masses, $\log T_{\text {eff }}, \log g$, and $\log L$ ), the constants of internal structure $\left(k_{2}, k_{3}\right.$, and $k_{4}$ ), the radius of gyration $\beta$, and the factor $\alpha$ that is related with the gravitational potential energy.
\end{abstract}

Key words. binaries: eclipsing - binaries: general - stars: evolution - stars: interiors - planetary systems

\section{Introduction}

Double-lined eclipsing binaries (DLEBS) are the main source of accurate, absolute dimensions of stars. In addition, some proximity effects can provide valuable information on the stellar interiors. In a close and eccentric binary system, the stellar configurations are distorted by tides and rotation. As a consequence of these distortions, there is a secular change of the position of the periastron. Such distortions can be described as a function of the internal structure of each star through the apsidal motion constants $\left(k_{2}, k_{3}\right.$, and $\left.k_{4}\right)$. For a complete description of the stellar distortions caused by tides and rotation, see Kopal $(1959,1978)$. As the apsidal motion period depends strongly on the relative radius $\left(r^{-5}\right)$, only systems with very accurate absolute dimensions can be used to perform the comparison between the theoretical and observed apsidal motion rates. An advantage of the apsidal motion rates is that they have been measured for a wide range of masses. Therefore, one can investigate the interior of the stars under very different physical conditions, thus apsidal motion is a good and complementary test for the theory of stellar evolution.

A systematic effect was detected when the theoretical apsidal motion constants were compared with the observed ones: the real stars seemed to be more mass concentrated than predicted by the theoretical stellar models. For a short historical review on this point, see Claret \& Giménez (1993). These authors, using

\footnotetext{
* Tables 2-55 are only available at the CDS via anonymous $\mathrm{ftp}$ to cdsarc.u-strasbg. fr (130.79.128.5) or via http://cdsarc. u-strasbg.fr/viz-bin/qcat?J/A+A/628/A29
}

several improvements made to the stellar models available at that time (mainly opacities), were able to somewhat reduce discrepancies for about 20 DLEBS. In 1997, analysing only relativistic eclipsing binaries, Claret (1997) showed that general relativity predictions were able to explain the shift in the periastron position without the need for an alternative theory of gravitation. The problematic case of DI Her was solved observationally through the Rossiter-McLaughlin effect some years later by Albrecht et al. (2009). A few months later, Claret et al. (2010) redetermined the apsidal motion rate for DI Her using new times of minima. Also new evolutionary models were adopted that were based on new absolute dimensions. With these data, these authors were able to reduce the difference between the theoretical and observed apsidal motion rate to only $10 \%$.

Another small step forward was achieved by Claret \& Giménez (2010). Using a larger sample of DLEBS (with improved absolute dimensions), they have shown that the discrepancies between the theoretical predictions and the observational rates of apsidal motion have decreased significantly. In that paper, in addition to the classical contribution to the apsidal motion rate because of static tides, dynamic tides were also considered due to the effects of the compressibility of the stellar fluid, mainly when the system is near the synchronism.

Since then, the accuracy of the absolute dimensions of the DLEBS have increased, which is the same for apsidal motion rates (Torres et al. 2010). The evolutionary stellar models have also improved (opacities, thermonuclear energy generation rates, equation of state, extra-mixing, etc.). On the other hand, the apsidal motion constants and the moment of inertia are also 
important in order to investigate the tidal evolution of close binary stars and exo-planetary systems (Hut 1981, 1982). Therefore, a new generation of stellar models is necessary to be compared with the recent observational data about absolute dimensions and apsidal motion rates of DLEBS as well as with the data from the exo-planetary systems. Such updated evolutionary models are the main aim of the present research. The paper is organised as follows. Section 2 is dedicated to the introduction of the differential equations used to compute the theoretical apsidal motion constants as well as the moment of inertia and gravitational potential energy. Section 3 is devoted to the analysis of the characteristics of the new models. Finally, Table 1 summarises the properties of the models available at the CDS (Centre de Données Astronomiques de Strasbourg) or those that come directly from the author.

\section{Stellar models, internal structure constants, and tidal evolution}

The stellar models were computed using the Modules for Experiments in Stellar Astrophysics package (MESA; Paxton et al. 2011, 2013, 2015) version 7385, which does not include the effects of rotation. The adopted mixing-length parameter $\alpha_{\text {MLT }}$ was 1.84 (the solar-calibrated value). However, it is expected that the $\alpha_{\text {MLT }}$ parameter depends on the evolutionary status and/or metallicity, according to the theoretical predictions of the 3D simulations (Magic et al. 2015). Due to the different input physics of MESA, mainly the equation of state and opacities, a direct comparison of the $\alpha_{\mathrm{MLT}}$ adopted here with those generated by $3 \mathrm{D}$ simulations is not straightforward. In all calculations, the microscopic diffusion was included. For the opacities, the adopted element mixture is that by Asplund et al. (2009). The helium content is given by the following enrichment law: $Y=0.249+1.67 \mathrm{Z}$. The covered mass range was from 0.8 up to $35.0 M_{\odot}$. For stars that are more massive than $12 M_{\odot}$, we adopted the scheme of mass loss from Vink et al. (2001) with a multiplicative scale factor $\eta=0.1$. This is not to be confused with $\eta$ of the Radau equation (see below). For less massive stars in the red giants branch, we adopted the formalism by Reimers (1977) with $\eta=0.2$. For AGB stars we adopted the formula given in Blocker (1995) with $\eta=0.1$. In this paper, convective core overshooting was introduced using the diffusive approximation, given by the free parameter $f_{o v}$ (Freytag et al. 1996; Herwig et al. 1997). In this approximation, the diffusion coefficient in the overshooting region is given by the expression $D_{o v}=D_{o} \exp \left(\frac{-2 z}{H_{v}}\right)$ and $D_{o}$ is the diffusion coefficient at the convective boundary, $z$ is the geometric distance from the edge of the convective zone, $\mathrm{H}_{v}$ is the velocity scale-height at the convective boundary expressed as $\mathrm{H}_{v}=f_{o v} \mathrm{H}_{p}$, and the coefficient $f_{o v}$ is a free parameter governing the width of the overshooting layer. It is known that models with core overshooting are more centrally concentrated in mass than their standard counterparts (Claret \& Giménez 1991). At that time, it was usual to take the overshooting parameter $\left(\alpha_{o v}\right)$ as a constant for the entire range of stellar masses. Instead, here, we adopted the relationship between the stellar mass and $\mathrm{f}_{o v}$ found by Claret \& Torres (2018) in their Fig. 3.

Typically, DBLES that show apsidal motion are not very evolved. Nonetheless, all models were followed from the premain-sequence (PMS) up to the first ascent giant branch or beyond. The tables containing the models begin at the zero-age main-sequence (ZAMS), defined here as the locus of the HR diagram at which the central hydrogen content drops to $99.4 \%$ of its initial value.
Table 1. Stellar models.

\begin{tabular}{|c|c|c|}
\hline Name & Initial mass (solar mass) & {$[\mathrm{Fe} / \mathrm{H}]$} \\
\hline Table2 & 0.80 & 0.00 \\
\hline Table 3 & 1.00 & 0.00 \\
\hline Table4 & 1.20 & 0.00 \\
\hline Table 5 & 1.40 & 0.00 \\
\hline Table6 & 1.60 & 0.00 \\
\hline Table7 & 1.80 & 0.00 \\
\hline Table8 & 2.00 & 0.00 \\
\hline Table9 & 2.50 & 0.00 \\
\hline Table10 & 3.00 & 0.00 \\
\hline Table11 & 5.00 & 0.00 \\
\hline Table12 & 7.00 & 0.00 \\
\hline Table13 & 10.00 & 0.00 \\
\hline Table14 & 12.00 & 0.00 \\
\hline Table15 & 15.00 & 0.00 \\
\hline Table16 & 20.00 & 0.00 \\
\hline Table17 & 25.00 & 0.00 \\
\hline Table18 & 30.00 & 0.00 \\
\hline Table19 & 35.00 & 0.00 \\
\hline Table20 & 0.80 & -0.50 \\
\hline Table21 & 1.00 & -0.50 \\
\hline Table 22 & 1.20 & -0.50 \\
\hline Table23 & 1.40 & -0.50 \\
\hline Table24 & 1.60 & -0.50 \\
\hline Table25 & 1.80 & -0.50 \\
\hline Table26 & 2.00 & -0.50 \\
\hline Table27 & 2.50 & -0.50 \\
\hline Table 28 & 3.00 & -0.50 \\
\hline Table29 & 5.00 & -0.50 \\
\hline Table30 & 7.00 & -0.50 \\
\hline Table31 & 10.00 & -0.50 \\
\hline Table 32 & 12.00 & -0.50 \\
\hline Table33 & 15.00 & -0.50 \\
\hline Table34 & 20.00 & -0.50 \\
\hline Table35 & 25.00 & -0.50 \\
\hline Table36 & 30.00 & -0.50 \\
\hline Table37 & 35.00 & -0.50 \\
\hline Table 38 & 0.80 & -1.00 \\
\hline Table39 & 1.00 & -1.00 \\
\hline Table40 & 1.20 & -1.00 \\
\hline Table41 & 1.40 & -1.00 \\
\hline Table42 & 1.60 & -1.00 \\
\hline Table43 & 1.80 & -1.00 \\
\hline Table44 & 2.00 & -1.00 \\
\hline Table45 & 2.50 & -1.00 \\
\hline Table46 & 3.00 & -1.00 \\
\hline Table47 & 5.00 & -1.00 \\
\hline Table48 & 7.00 & -1.00 \\
\hline Table49 & 10.00 & -1.00 \\
\hline Table50 & 12.00 & -1.00 \\
\hline Table51 & 15.00 & -1.00 \\
\hline Table52 & 20.00 & -1.00 \\
\hline Table53 & 25.00 & -1.00 \\
\hline Table 54 & 30.00 & -1.00 \\
\hline Table55 & 35.00 & -1.00 \\
\hline
\end{tabular}

A significant observational advance in apsidal motion studies was recently achieved by Zasche \& Wolf (2019) who investigated 21 eccentric eclipsing binaries located in the small Magellanic Cloud. Taking this into account, we decided to extend 
the calculations to the following three metallicities: $[\mathrm{Fe} / \mathrm{H}]=$ $0.00,-0.50$, and -1.00 . This allows analysis of interpolating in the three grids in order to study DLEBS showing apsidal motion and/or tidal evolution that are also in less metallic environments.

The internal structure constants $-k_{2}, k_{3}$, and $k_{4}-$ are derived by integrating the Radau equation

$\frac{a \mathrm{~d} \eta_{j}}{\mathrm{~d} a}+\frac{6 \rho(a)}{\bar{\rho}(a)}\left(\eta_{j}+1\right)+\eta_{j}\left(\eta_{j}-1\right)=j(j+1), j=2,3,4$

where,

$\eta \equiv \frac{a}{\epsilon_{j}} \frac{\mathrm{d} \epsilon_{j}}{\mathrm{~d} a}$

$a$ is the mean radius of the stellar configuration, $\epsilon_{j}$ is a measure of the deviation from sphericity, $\rho(a)$ is the mass density at the distance $a$ from the centre, and $\bar{\rho}(a)$ is the mean mass density within a sphere of radius $a$.

The resulting apsidal motion constant of order $j$ is given by

$k_{j}=\frac{j+1-\eta_{j}(R)}{2\left(j+\eta_{j}(R)\right)}$

where $R$ indicates the values of $\eta_{j}$ at the surface of the star.

We note that these equations are derived in the framework of static tides. For the case of dynamic tides, more elaborated equations are necessary. The rate of classical apsidal motion was derived assuming that the orbital period is larger than the periods of the free oscillation modes. However, dynamic tides can significantly impact the theoretical predictions based on static tides. This is mainly due to the effects of the compressibility of the stellar fluid if the systems are near synchronism. In this case, for higher rotational angular velocities, additional deviations caused by resonances will appear if the forcing frequencies of the dynamic tides come into range of the free oscillation modes of the component stars. For numerical details on the impact of the dynamical tides see Claret \& Willems (2002), Willems \& Claret (2003), Claret \& Giménez (2010).

As previously mentioned, the present models were computed without taking rotation into account. To evaluate these effects, a correction on the internal structure constants was introduced by Claret (1999) which is given by $\Delta \log k_{2} \equiv \log k_{2, \text { stan }}-\lambda$, where $\lambda=2 V^{2} /(3 g R), g$ is the surface gravity, and $V$ is the equatorial rotational velocity.

The effects of general relativity on the moment of inertia and gravitational potential energy are not so important for stars at the PMS, main-sequence (MS), or for white dwarfs (WD). However, for consistency with our previous papers on compact stars, the relativistic formalism is adopted throughout. The moment of inertia is given by the following equation, where $\beta$ is the radius of gyration:

$J=\frac{8 \pi}{3} \int_{0}^{R} \Lambda(r) r^{4}\left[\rho^{\prime}(r)+P(r) / c^{2}\right] \mathrm{d} r$,

$I \approx \frac{J}{\left(1+\frac{2 G J}{R^{3} c^{2}}\right)} \equiv(\beta R)^{2} M$.

On the other hand, the amount of work necessary to bring the entire spherical star in from infinity is given by

$\Omega=-4 \pi \int_{0}^{R} r^{2} \rho^{\prime}(r)\left[\Lambda^{1 / 2}(r)-1\right] \mathrm{d} r \equiv-\alpha \frac{G M^{2}}{R}$, where $P(r)$ is the pressure, $\rho^{\prime}(r)$ the energy density, and the auxiliary function, $\Lambda(r)$, is given by $\left[1-\frac{2 G m(r)}{r c^{2}}\right]^{-1}$. The parameter $\alpha$ is a dimensionless number that measures the relative mass concentration. For example, for polytropes, we have $\alpha=3 /(5-n)$, where $n$ is the polytropic index.

Equations (1), (4), and (5) were integrated simultaneously through a fifth-order Runge-Kutta method, with a tolerance level of $10^{-7}$.

The factors $\alpha$ and $\beta$ show very interesting behaviour. Defining a new function as

$\Gamma \equiv \frac{[\alpha \beta]}{\Lambda(R)^{0.8}}$

Claret \& Hempel (2013) computed a series of stellar models from the PMS up to the white dwarf cooling sequences. They found a connection between the large variations of $\Gamma$ during the intermediary evolutionary phases and the specific nuclear power. A threshold for the specific nuclear power was also found. Below this limit, $\Gamma$ is invariant $(\approx 0.4)$ and recovers the initial value at the PMS.

Other final products of the stellar evolution - neutron and quark stars - for which the effects of general relativity are strong, also present this invariance that is independent of the mass and equation of state. Furthermore, by using a core-collapse supernova simulation, the PMS value of $\Gamma$ is recovered at the onset of the formation of a black hole. Therefore, regardless of the final products of the stellar evolution; white dwarfs; neutron, hybrid, and quark stars; or proto-neutron stars at the onset of the formation of a black hole, it can be concluded that they present a "memory effect" and recover the fossil value of $\Gamma \approx 0.40$ that is acquired during the PMS (Claret 2014). The invariance of $\Gamma$ was also extended to another category of celestial bodies, the gaseous planets, with masses ranging from 0.1 to $50 M_{J}$ (Claret \& Hempel 2013). Such models were followed from the gravitational contraction up to to $\approx 20 \mathrm{Gyr}$. In addition, a macroscopic stability criterion for neutron and quark star models, based on the properties of the relativistic product $\alpha \beta$, was introduced.

The connection between the factors, $\alpha$ and $\beta$, with the Jacobi virial theorem is clear for conservative systems recalling that such an equation can be written as

$\frac{\partial^{2} \phi}{\partial t^{2}}=\Omega+2 T=2 E_{\mathrm{T}}-\Omega$

In the above equation, the Jacobi function is given by $\phi=$ $1 / 2 \sum_{i} m_{\mathrm{i}}\left(x_{\mathrm{i}}^{2}+y_{\mathrm{i}}^{2}+z_{\mathrm{i}}^{2}\right), T$ is the kinetic energy and $E_{\mathrm{T}}$ is the total energy. In the case of spherical symmetric configurations, $\phi=3 / 4 I \equiv 3 / 4 M(R \beta)^{2}$. For conservative systems, Eq. (7) can be transformed in a differential equation with only one unknown function. An important question coming from the resulting equation is whether the product $\alpha \beta$ is constant for all stellar evolutionary phases. However, as shown in the above mentioned papers, only during the PMS and the earlier stages of the main sequence is the product, $\alpha \beta$, almost constant. When the stellar models evolve out of the main sequence, this product increases during the later phases before reaching the compact star stage. In this stage, the product recovers its original value $(\approx 0.40)$, acquired at the PMS. For an insightful vision of the virial theorem, see Collins (2003). For a more detailed discussion on the relation of the Jacobi differential equation and stellar evolution, see Sect. 4 by Claret (2012). 


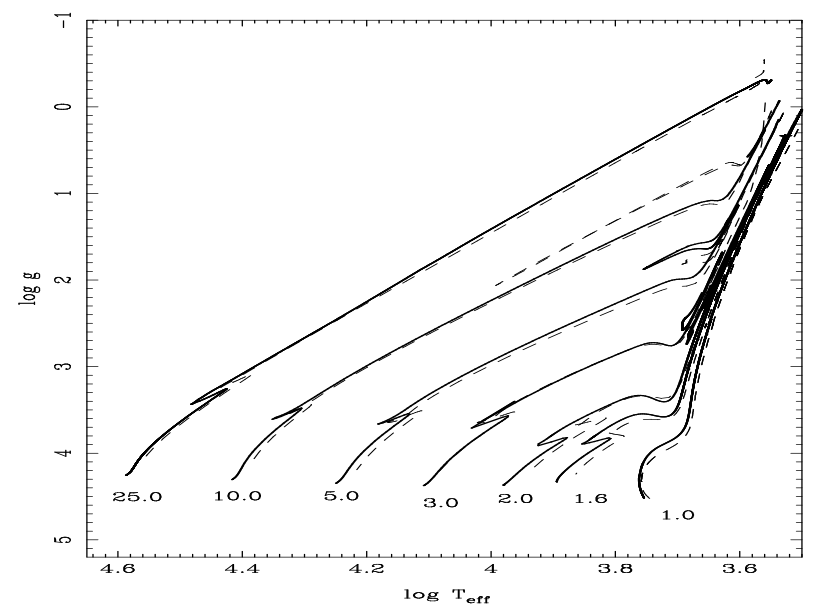

Fig. 1. HR diagram for some selected models. The masses are indicated in solar units. Continuous lines represent the present models while dashed ones denote the models by Claret (2004). Solar composition.

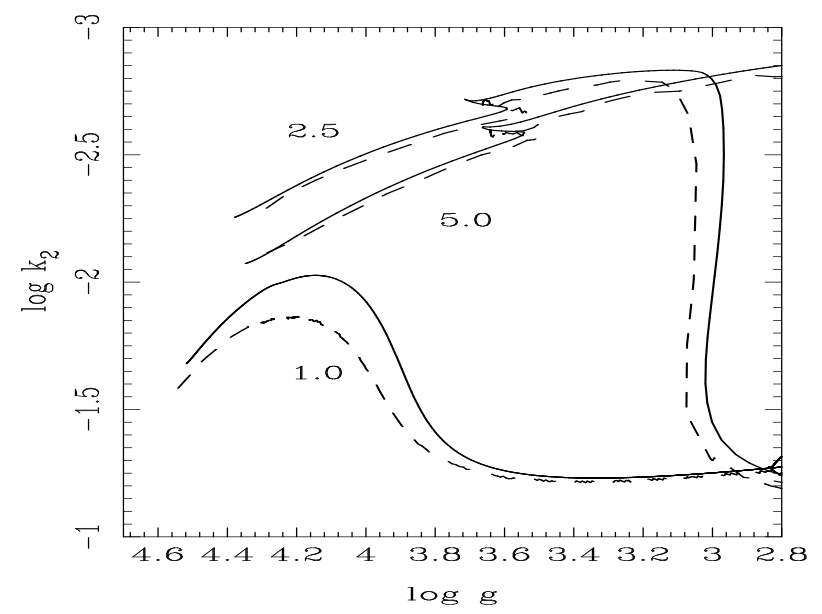

Fig. 2. Apsidal motion constant of order two for new and old models. The masses, in solar units, are indicated by numbers. Same captions as in Fig. 1.

\section{Tidal evolution constants: The apsidal motion and the moment of inertia}

Returning to the main purpose of the paper (the constants of internal structure), we adopted some selected models by Claret (2004) for comparison purposes. The 2004 models were computed for a slightly different chemical composition $(X=$ $0.700, Z=0.02$, based on the Grevesse \& Sauval 1998) mixture and a different $\alpha_{\mathrm{MLT}}=1.68$.

Figure 1 shows the HR diagram for the new and old models. There are two systematic effects. Firstly, the new models present higher effective temperatures than their old counter-parts. Secondly, the radii of the new models are smaller than the old ones, and the effect is smaller for low-mass stars.

Concerning the theoretical apsidal motion constants, in general, the new models are more concentrated in mass than the old ones (Fig. 2). However, the differences are not constant and progressively decrease from less massive to more massive models. Considering, for example, models of $1.00 M_{\odot}$, the difference is of the order of 0.20 dex (in the logarithmic scale) in the middle of the main sequence, which is of the order of the mean observational errors. For the more massive models (e.g. 2.50 and $5.00 M_{\odot}$ ), the differences between the new models and the old

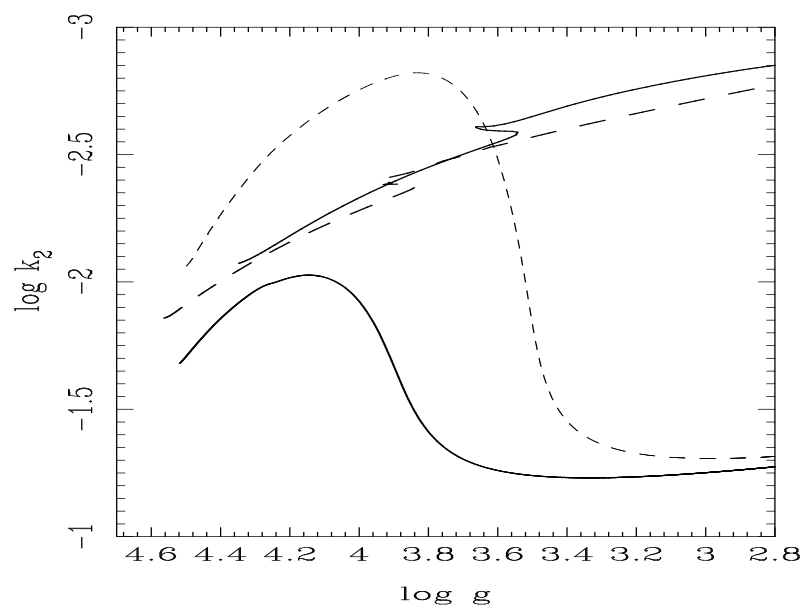

Fig. 3. Apsidal motion constant of order two for present models. Computed for $[\mathrm{Fe} / \mathrm{H}]=0.00$ (continuous lines) and for $[\mathrm{Fe} / \mathrm{H}]=-1.0$ (dashed lines). Models with 1.00 and $5.00 M_{\odot}$.

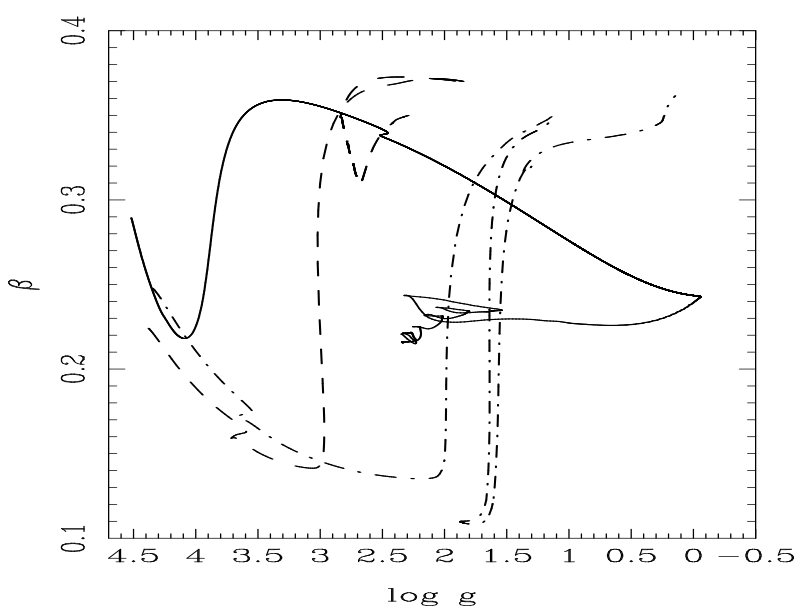

Fig. 4. The evolution of radius of gyration for some selected models. Continuous line represents the model with $1.00 M_{\odot}$, the dashed and dashed-point lines denote the models with 2.5 and $5.0 M_{\odot}$, respectively. Solar composition.

models are only of the order of 0.05 dex, though they are still important.

The differences between the two sets of models discussed above have diverse causes, such as the adopted values of $\alpha_{\mathrm{MLT}}$ (1.84 and 1.68). The equation of state also contributes. It is important to note that this is mainly the case for the less massive models. The rates of mass loss, which are different from those adopted in the 2004 models, also influence both the shape of the tracks in the HR diagram and their internal structure. Another reason for the differences in the 2004 models is that a constant value for the amount of core overshooting was adopted for all masses; conversely, as previously mentioned, we introduced a dependence on the stellar mass for the core overshooting as given by Claret \& Torres (2018).

The effects of the metallicity on the internal structure is shown in Fig. 3 for models with 1.00 and $5.00 M_{\odot}$ for $[\mathrm{Fe} / \mathrm{H}=$ $0.00]$ with continuous lines, and for $[\mathrm{Fe} / \mathrm{H}=-1.00]$ with dashed lines. The differences between the $[\mathrm{Fe} / \mathrm{H}=0.00]$ and $[\mathrm{Fe} / \mathrm{H}=-1.00]$ models are important. This mainly concerns less massive models during the main sequence and can achieve up to 0.80 dex for the $1.00 M_{\odot}$ models. These differences, in general, decrease with the stellar mass. 


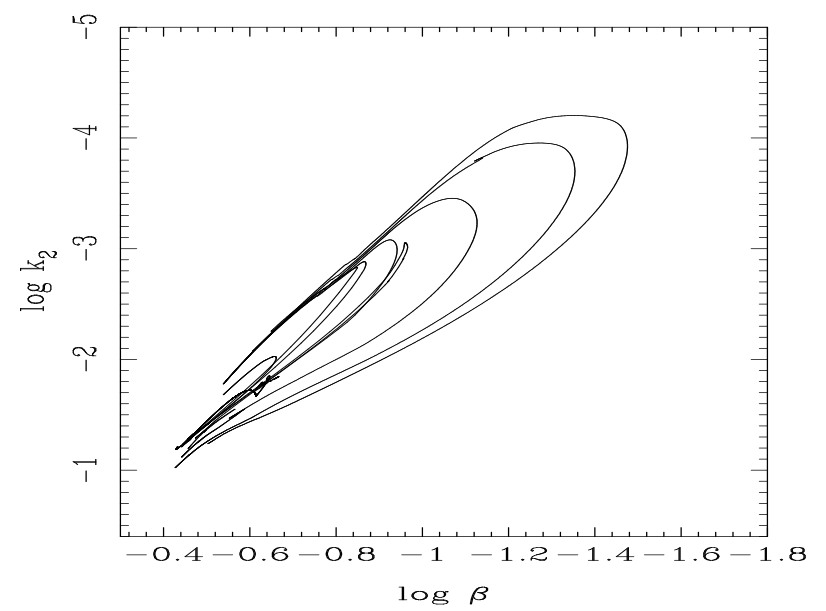

Fig. 5. Relationship between evolving $\log \beta$ and $\log k_{2}$ for models with $1.00,2.50,5.00,10.00,15.00,20.00$, and $25.00 M_{\odot}$. Solar composition.

For homogeneous models, the radius of gyration shows a different behaviour for masses larger or smaller than $\approx 1.5 M_{\odot}$, that is there is a minimum of $\beta$ around this value. Such behaviour is connected with the change in the dominant source of thermonuclear energy from the pp chain to the CNO cycle (Fig. 2 by Claret \& Giménez 1989). In Fig. 4, we show the evolution of $\beta$ for models of $1.00,2.50$, and $5.00 M_{\odot}$. When the star evolves, the behaviour of $\beta$ becomes more complex, showing some loops for advanced evolutionary phases. Despite the complex behaviour of the apsidal motion constant of order two and the radius of gyration as a function of $\log g$ shown in Figs. 2 and 4, both have an approximately linear relationship that is independent of their evolution for a wide range of stellar masses. The resulting lobe shown in Fig. 5 is due to nuclear reactions and chemical inhomogeneities which lead to structural changes. The aspect of Fig. 5 is not surprising given that $\log k_{2}$ and $\log \beta$ give a measure of the mass concentration for each evolutionary stage of the models through the integration of the respective differential equations of second and first order, respectively.

Finally, Table 1 summarises the data available at the CDS or directly from the author. Additional calculations for other chemical compositions and/or for specific masses can be performed upon request.

Acknowledgements. I am grateful to the anonymous referee for the careful reading of the paper and for her/his helpful suggestions. I am grateful to B. Rufino, G. Torres, and V. Costa for their useful comments. The Spanish MEC (AYA2015-71718-R and ESP2017-87676-C5-2-R) is gratefully acknowledged for its support during the development of this work. AC also acknowledges financial support from the State Agency for Research of the Spanish MCIU through the Center of Excellence Severo Ochoa award for the Instituto de Astrofísica de Andalucía (SEV-2017-0709). This research has made use of the SIMBAD database, operated at the CDS, Strasbourg, France, and of NASA's Astrophysics Data System Abstract Service.

\section{References}

Albrecht, S., Reffert, S., Snellen, I. A. G., \& Winn, J. N. 2009, Nature, 461, 373 Asplund, M., Grevesse, N., Sauval, A. J., \& Scott, P. 2009, ARA\&A, 47, 481

Blocker, T. 1995, A\&A, 297, 727

Claret, A. 1997, A\&A, 327, 11

Claret, A. 1999, A\&A, 350, 56

Claret, A. 2004, A\&A, 424, 919

Claret, A. 2012, A\&A, 543, A67

Claret, A. 2014, A\&A, 562, A31

Claret, A., \& Hempel, M. 2013, A\&A, 552, A29

Claret, A., \& Giménez, A. 1989, A\&AS, 81, 37

Claret, A., \& Giménez, A. 1991, A\&A, 244, 319

Claret, A., \& Giménez, A. 1993, A\&A, 277, 487

Claret, A., \& Giménez, A. 2010, A\&A, 519, A57

Claret, A., \& Torres, G. 2018, ApJ, 859, 100

Claret, A., \& Willems, B. 2002, A\&A, 388, 518

Claret, A., Torres, G., \& Wolf, M. 2010, A\&A, 515, A4

Collins, II., G. W. 2003, The Virial Theorem in Stellar Astrophysics, Web Edition Freytag, B., Ludwig, H.-G., \& Steffen, M. 1996, A\&A, 313, 497

Grevesse, N., \& Sauval, A. J. 1998, Space Sci. Rev., 85, 161

Herwig, F., Bloecker, T., Schoenberner, D., \& El Eid, M. 1997, A\&A, 324, L81

Hut, P. 1981, A\&A, 99, 126

Hut, P. 1982, A\&A, 102, 37

Kopal, Z. 1959, Close Binary Systems (London: Chapman and Hall)

Kopal, Z. 1978, Dynamics of Close Binary Systems (Dordrecht, Holland: Reidel)

Magic, Z., Weiss, A., \& Asplund, M. 2015, A\&A, 573, A89

Paxton, B., Bildsten, L., Dotter, A., et al. 2011, ApJS, 192, 3

Paxton, B., Cantiello, M., Arras, P., et al. 2013, ApJS, 208, 4

Paxton, B., Marchant, P., Schwab, J., et al. 2015, ApJS, 220, 15

Reimers, D. 1977, A\&A, 61, 217

Torres, G., Andersen, J., \& Giménez, A. 2010, A\&ARv, 18, 67

Vink, J. S., de Koter, A., \& Lamers, H. J. G. L. M. 2001, A\&A, 369, 574

Willems, B., \& Claret, A. 2003, A\&A, 410, 289

Zasche, P., \& Wolf, M. 2019, AJ, 157, 87 